\title{
Automated cyclers used in peritoneal dialysis: technical aspects for the clinician
}

This article was published in the following Dove Press journal:

Medical Devices: Evidence and Research

23 January 2015

Number of times this article has been viewed

\section{Rafia I Chaudhry \\ Thomas A Golper \\ Division of Nephrology and Hypertension, Vanderbilt Center for Kidney Disease, Vanderbilt University Medical Center, Nashville, TN, USA}

Correspondence: Thomas A Golper Vanderbilt University Medical Center, S-3303 Medical Center North, II6I 2 I st Avenue South, Nashville, TN 37232-2372, USA

$\mathrm{Tel}+\mathrm{I} 6153432220$

Email thomas.golper@vanderbilt.edu

\begin{abstract}
Peritoneal dialysis (PD) is a widely accepted and increasingly popular form of dialysis. The invention and technological advancement of the PD cycler further makes PD a convenient option. Prescription-specific parameters are entered into the cycler, which then automatically carries out the steps involved in continuous cycling PD. We review the basics, technical aspects, challenges, and advancements of the cycler.
\end{abstract}

Keywords: peritoneal dialysis, automated peritoneal dialysis, cycler peritoneal dialysis

\section{Introduction}

The concept of peritoneal dialysis (PD) first surfaced in the late 1800s, but George Ganter was the first scientist to formally publish the trials of PD, as reviewed by Palmer. ${ }^{1,2}$ Although initially considered inferior to hemodialysis (HD), PD has evolved remarkably over the decades proving itself to be a competitive alternative to HD. Now, PD is also recognized to offer some advantages over HD, including conserving dialysis access options and the convenience of performing dialysis overnight at home.

PD is now a widely accepted, effective form of renal replacement therapy for patients with end-stage renal disease. There are different methods of performing PD, which are broadly divided into continuous cycling PD (CCPD), also referred to as automated PD (APD), using the cycler, and continuous ambulatory PD (CAPD), performed without a machine, ie, patients performing the entire therapy manually. Expert opinion concludes that around $60 \%$ of patients on PD in the US opt for CCPD or APD, compared to approximately $50 \%$ of PD patients in Europe who opt CCPD or APD. ${ }^{3}$ The remaining patients opt for CAPD. With the rise in popularity of the cycler, the percentage of patients on CCPD is trending upward.

The institution of the cycler appears to correlate with the rise in popularity of PD. One of the reasons for this may be the convenience of performing dialysis overnight while asleep.

Most of the earlier cyclers were modeled after Lasker's, and the initial PD cycler was developed in Seattle, 1962. ${ }^{4,5}$ PD cyclers have evolved since that time with rapidly advancing technology. At present, there are currently two major companies, Baxter and Fresenius, that manufacture PD cyclers in the US and enable this form of dialysis to be available countrywide.

Because our experience is limited to the US, here we review the basic mechanism, evolution, use, and expert opinion on the PD cyclers available in the US. 


\section{Peritoneal dialysis basics}

A catheter inserted through the patient's abdomen allows the peritoneal cavity to be drained; the dialysate solution is then infused into the peritoneal cavity and allowed to dwell for the exchange of electrolytes and waste products between the solution and patient's blood, via diffusion and convection. After a few hours, the solution is again drained, and is now called the "effluent", which is composed of the dialysate solution at the end of the exchange, waste products including urea and creatinine among others, and any ultrafiltrate. The method by which these steps (ie, fill, dwell, and drain) are performed is broadly categorized as PD.

For CAPD, the patient infuses dialysate solution by hanging the solution bag on a pole, which allows gravity to direct the solution into the peritoneal cavity at the beginning of each cycle. Similarly, at the end of the dwell period, the effluent dialysate is drained via gravity to the effluent bag, thus completing the cycle, which constitutes one exchange. This is in contrast to CCPD (APD), where a cycler is connected to the patient via tubing and programmed to perform a certain number of cycles over a period of time. Variables such as the inflow volume, dwell time, number of exchanges, and duration of each exchange, along with other parameters are programmed into the cycler customization for each patient. During nocturnal CCPD, the patient remains connected to the cycler for the entire therapy. However, the patient also uses the cycler to perform "walk-aways" or "pauses", which implies that the cycler is used to drain dialysate and infuse the solution, but during the dwell phase, the patient is disconnected from the cycler to allow freedom of movement.

The process of starting an individual on PD begins with clinical training for the patient and possible partners. Each PD center will have its own protocol. On average, this involves training session over 2 weeks, where qualified PD nurses will work with patients to assist them in becoming independent and comfortable in carrying out their dialysis using the cycler at home. These training sessions end only when both the nurse and patient feel comfortable and confident that the patient can handle and troubleshoot the cycler at home, independently, in a safe manner. The supervising nephrologist has final authority over this process.

\section{Mechanics of the PD cycler}

Current cyclers' technology delivers solution and removes dialysate from the patient. The goal of the cycler is to provide safe and effective dialysis in an automated manner, primarily while the patient is asleep, making it a more convenient option.
For this to happen, the cycler must incorporate volumetric precision and timing to coordinate the infusion volume, rate of dialysate flow, dwell time, drain time, and last fill volume before being disconnected, and monitor with safety alarms to alert the system in case of a problem. The cycler also has to monitor the amount of ultrafiltration (UF) with each exchange to ensure that the dialysate is draining appropriately. Current cyclers record the amount of UF by subtracting the total fill volume from the total drain volume.

Cycler components include:

- heater,

- cassette with chambers and valves,

- dialysate bag, and

- drain bag (15 L) or drain into commode.

The cassette is loaded into the cycler. During the initial phase or "priming", fresh solution is drawn into the empty heater bag, where it is warmed to body temperature, before being directed to the cassette via the patient line. ${ }^{6}$ Similarly, during the drain cycle, the fluid is sent into the disposable cassette. In both infusion and drain phases, the cassette and pumping technologies measure dialysate volume (see "Technical aspects of current cyclers" section) to provide the programmed fill volume during the infusion phase or to calculate the amount of ultrafiltrate during the drain phase. The solution is then redirected appropriately by opening and closing different valves to its destination, ie, either the peritoneal cavity (fill phase) or the effluent bag (drain phase). ${ }^{6}$

In case of a power failure, all valves will close to prevent any dialysate flow. If power returns within 2 hours, the cycler automatically restarts. However, if power is interrupted for a longer period, then the patient is alerted with an alarm, and will have to perform a manual drain of the dialysate in the peritoneal cavity.

Each time a patient connects to the cycler to initiate the therapy, the cycler begins with an initial drain (I-Drain) to empty the peritoneal cavity first. The automated cycler then coordinates the three main phases of PD, ie, fill, dwell, and drain, which constitute a "cycle". The volume of solution infused is individualized and programmed into the cycler, along with the number of cycles and duration of therapy (time spent connected to the cycler). The cycler is able to calculate the fill time, drain time, minimal flow rate based on the specifications for the machine, and appropriately sound alarms or warnings if these are not met. ${ }^{7,8}$

Both Baxter and Fresenius now offer models that feature a digital card (see Pro Card in "Technical aspects of current cyclers" section) that is able to store therapy information for the last 30-90 days, depending on the model and digital 
card capacity. The patient can take this to their monthly dialysis visit where the therapy details can be downloaded by the staff.

We envision a future application where this information might be transmitted remotely to the home dialysis center.

\section{Modes of PD offered by the automated cycler}

There are many types of APD in practice. Here, we highlight some popular modes: $: 10$

1. CCPD,

2. intermittent peritoneal dialysis (IPD) and nocturnal intermittent peritoneal dialysis (NIPD),

3. tidal peritoneal dialysis (TPD),

4. Hi-Dose CCPD, and

5. Hi-Dose TPD.

CCPD: It is a form of continuous therapy where APD is performed by the cycler while the patient is asleep. Patients have fluid in their peritoneal cavity at all times during the therapy while the patient is attached to the cycler, except for the end of the drain period before refilling begins, and fluid remains in the peritoneal cavity after disconnecting. (This definition is used by the Liberty cycler by Fresenius, while the HomeChoice and HomeChoice Pro by Baxter use CCPD to define therapy that offers the patient the option to be wet or dry during the day, disconnected from the cycler).

IPD: Patients dialyze intermittently for a few days a week and finish each intermittent period by draining completely. These are usually patients who were recently initiated on PD and those with residual kidney function, which allows them to get adequate clearance with a smaller dose of dialysis. A variety of this is NIPD, where nocturnal cycling occurs with a dry day, every night.

TPD: This involves the exchange of a certain volume percentage or absolute volume during each cycle, leaving a residual amount in the peritoneal cavity at most times, through the infusion and drain phases. One advantage of this mode is the possibility of reducing the incidence of "drain pain", which we discuss later. The minimum target ultrafiltrate is programmed into the cycler to minimize the risk of overfill or increased intraperitoneal volume.

Notably, TPD can be overlapped with any other forms of PD, ie, CCPD, CAPD, and IPD.

Hi-Dose CCPD and TPD are specific terminologies used by Baxter to describe the practice of adding daytime cycles to traditionally nocturnal therapies such as NIPD and TPD to provide increased dialysis dose to patients. ${ }^{6,9}$ Patients using other cyclers may also be prescribed therapy in this manner to provide additional dialysis. The "walk-away" or pauses referred to earlier apply to the Hi-Dose CCPD.

All modes can also be further classified based on fill volume, ie, standard fill mode or low fill mode. Most adult patients will use standard fill modes (fill volume $>1,000 \mathrm{~mL}$ ); while low fill modes are available for pediatric patients and for adult patients weighing less than $45 \mathrm{~kg}$ requiring $<1,000 \mathrm{~mL}$ fill volume, when hernias or leaks are present, or after certain surgeries. ${ }^{5,6}$ The Fresenius Liberty models only offer a minimal fill volume of $500 \mathrm{~mL}$; pediatric patients requiring less than this use the Baxter HomeChoice cycler, which allows fill volumes ranging from $60 \mathrm{~mL}$ to $1,000 \mathrm{~mL} .^{6,10}$

\section{Technical aspects of current cyclers}

All details below are pertinent to the Baxter cyclers Home Choice and Home Choice Pro. ${ }^{6,9}$ For logistic reasons, we were unable to obtain similar details on the Fresenius cyclers.

Average size and models of current cyclers in use:

- HomeChoice - 7 inches $\times 19.5 \times 15.7$ inches, $26.8 \mathrm{lbs}$

- HomeChoice Pro -7 inches $\times 19.5 \times 15.7$ inches, $26.8 \mathrm{lbs}$

- Fresenius Liberty cycler with tabletop design

Basic components of the cycler system:

- solution bags,

- disposable set (standard set for fill volume $>1,000 \mathrm{~mL}$ and low recirculation volume set for fill volumes $<1,000 \mathrm{~mL}$ ),

- drain bag or drain line extension,

- disconnect caps, and

- patient line extension.

Patients are educated in one way or another to check the integrity of the solution bags. One method for example is using the mnemonic SEAL, ie, strength, expiration date, amount, and leaks. The bag is then placed on the heater tray. The cycler is then switched on and step-by-step instructions were provided to the patient to start the therapy. The patient is asked to load the disposable set, ensuring that all clamps are initially closed and the cycler door is opened to load the cassette into the cycler.

The cycler provides the patient with two Drain Options, a Drain Line Extension or $15 \mathrm{~L}$ drain bag. If the patient's therapy requires more than $15 \mathrm{~L}$ drainage for the entire therapy, then they can use a "drain manifold" to connect two bags. Whichever the patient opts for they will attach it to the system. Lastly, the patient will be asked to connect the solution bags using sterile techniques. The "red line clamp" from the disposable set is connected to the heater bag. The solution bag on the heater pan must cover the heater sensor button. 
The "blue line clamp" is for the Last Fill Bag and the "white line clamps" are attached to the additional solution bags. The solution bag clamps are now opened, followed by the clamp on the patient line. The disposable set is "primed" to make sure that the fluid level is near the connector, at which point the patient connects to the cycler, again using proper sterile techniques.

All solutions moved by the system pass through the cassette. The system applies negative air pressure to the soft plastic membrane and pulls solution into the cassette chamber. The system then applies positive air pressure to the soft plastic membrane and pushes solution out of the chamber. The system applies pressure alternatively to the opposite ends of the chamber so that the solution continues to flow. This means that if negative pressure is applied to the right side to pull the solution into the chamber, positive pressure is applied to the left, pushing the solution to the appropriate destination. ${ }^{6}$

However, for moving solutions to and from the patient, the system uses pneumatic forces. The pump pressures generated by the system are on average set at $\pm 10.3 \mathrm{kPa}$ or $77.6 \mathrm{mmHg} .{ }^{6}$ When there is no fluid flow, the maximum pressure can increase to $\pm 15.2 \mathrm{kPa}$, ie, $113.8 \mathrm{mmHg}$ for a period of 10 seconds at the most. ${ }^{6}$

The system measures volume of the solutions using the Ideal Gas Equation principle. It monitors the pressure in the chamber area, and uses it to calculate the volume of air after it pushes the solution through one stroke. The volume of air then estimates the volume of solution moved. ${ }^{6}$ However, the system cannot differentiate between air in the cassette and air that came from the peritoneal cavity, or air that may have been trapped in the solution, which can result in volumetric errors in calculations under these circumstances.

There is no set rate for dialysate solution draining. HomeChoice assumes a default flow rate of $125 \mathrm{~mL} / \mathrm{min}$ for calculation of therapy specifics (eg, dwell time). However, it then also calculates the actual drain rate, monitoring for slow flow and low flow. Slow flow is defined as flow rate $<50 \mathrm{~mL} / \mathrm{min}$ (for standard fill mode) and $<15 \mathrm{~mL} / \mathrm{min}$ (low fill mode), while no flow is defined as flow rates $<12 \mathrm{~mL} / \mathrm{min}$ (for standard fill mode) and $<3 \mathrm{~mL} / \mathrm{min}$ (for low fill mode). These cutoffs are some of the parameters that prompt the low drain volume alarm and negative UF alarm to sound, as appropriate. The system incorporates drain logic, integrating all this information prior to sounding alarms; for example, it will allow slow flow to continue without sounding the alarm if the minimum drain time has not elapsed. ${ }^{6,9}$
Other standard estimated flow rates used by the system when calculating the number of cycles and dwell time include a fill rate of $220 \mathrm{~mL} / \mathrm{min}$, rate for refilling the heater bag as $300 \mathrm{~mL} / \mathrm{min}$, and drain rate as specified earlier. If the appropriate functions on the cycler are enabled (ie, adjust dwell up and adjust dwell down), the system will calculate the therapy parameters using the actual fill and drain rates from the previous cycle..$^{6,9}$

Solution temperatures are monitored by a solution temperature protective system, which uses a microprocessor, temperature sensor, and cutoff switch. This protective system senses temperature accurately to $\pm 2{ }^{\circ} \mathrm{C}$. An alarm is sounded within 5 seconds of sensing an overheated condition. ${ }^{6,9}$

In an attempt to maximize safety, the system continuously monitors various parameters including fluid volumes and flow rates, and alarms in case of an issue. In this situation, the cycler may at times stop moving the solutions and wait for the problem to be resolved by the user. It will display the type of alarm and may also record it on the Pro Card, if used.

In case of a power failure, or if the power switch is switched off during therapy, the cycler closes the occluder. If power returns within 30 minutes, the system will resume therapy without alarming. This is particularly helpful as patients may continue to have uninterrupted sleep if performing therapy at night. However, if more than 30 minutes lapse, the cycler alarms. If power is restored within 2 hours, therapy can be restarted; if longer than 2 hours have elapsed, then the therapy must be ended and restarted from the beginning. The patient can perform CAPD/manual drains and exchanges at any point. ${ }^{6,9}$

The Baxter HomeChoice Pro features a Pro Card, which is a computerized data transfer feature, which was first introduced in 1999. The Pro Card holds treatment information and can also be used by the dialysis center to automatically program a patient's therapy. The Pro Card information may also be transferred through to the dialysis center using a modem and phone line system, but this requires some specialized processes that are not yet universally utilized.

\section{Expert opinion on practical aspects of cycler use}

We conducted a questionnaire-based interview of PD nurses from (six) major PD centers, which included, Vanderbilt University Medical Center, Veterans Affairs Medical Center Nashville, University of Alabama at Birmingham, University Health Network Toronto, University of Pennsylvania, and Wake Forest University, to better understand the practical aspects of cycler use. We interviewed a total of 18 nurses from 
these six centers (Supplementary material). The comments were not necessarily amenable to tabulation, so we have collected and interpreted them for the purpose of this paper. We focused on addressing the benefits and challenges of the cycler in the opinion of nursing staff. We have attempted to categorize these responses based on similarities and will highlight the key areas.

\section{Increased intra-peritoneal volume or overfill and drain pain}

Overfill or increased intraperitoneal volume is a complication inherent to the current modes of operation of the PD cycler, and not specific or limited to a particular model, manufacturer, or technique. Overfill refers to excessive solution in the peritoneal cavity, beyond the target volume, or PD prescription fill volume. ${ }^{7,8}$ Cycler models for both Fresenius and Baxter are programmed to detect overfill by a number of algorithms and will alarm if they detect risk of increased intraperitoneal volume.

Some of the factors that increase the risk of overfill may be patient dependent, ie, the manner in which the patient performs therapy, and others are more mechanical. If a patient's prescription has a "last fill", where they finish their overnight treatments leaving a solution dwell that remains throughout the day, this must be drained prior to starting the next infusion.

Both Fresenius and Baxter cyclers now have a built in feature of the I-Drain to avoid the problem of increased intraperitoneal volume or overfill, and will attempt to drain the patient completely prior to infusing any dialysate into the peritoneal cavity. The cycler does not allow the patient to bypass the I-Drain under normal circumstances, and while this attempts to help in avoiding increased intraperitoneal volume, unfortunately it also leads to a lot of anxiety among patients who can experience significant I-Drain pain when the cycler may try to empty an already dry peritoneal cavity, irritating and tugging on the peritoneal membrane. This is because cyclers use pneumatic forces to pull fluid from the patient, as opposed to the conventional manual exchanges where dialysate flows out into the drain line by gravity. We received an overwhelming response from the nursing staff emphasizing the infrequently acknowledged issue of the I-Drain causing pain in a "dry" patient. Drain pain can occur as a consequence of a siphoning effect of draining fluid where sensitive intra-abdominal tissue (eg, bowel wall, omentum, bladder wall, fallopian tubes, uterine wall, etc) is sucked up against the PD catheter. ${ }^{7}$

The I-Drain may not be bypassed in the Baxter cyclers unless the Low Flow or No Flow condition is detected.
However, some of our staff helped clarify that the Baxter cycler does allow the I-Drain alarm to be set to $0 \mathrm{~mL}$, in which case it will allow the patient to avoid this issue and the alarm will not sound for Low Flow Volume or Drain. It is key to ensure that the I-Drain alarm is set appropriately. If set too low, the patient can experience problems of increased intraperitoneal volume, with dialysate remaining in the peritoneal cavity, and if it is set too high, the patient may experience drain pain. The I-Drain is calculated as a percentage of the last fill volume, usually programmed to a minimum of $70 \%-85 \% .^{9}$ The default I-Drain alarm for Standard Fill Mode in Baxter cyclers is set at the greatest of $1,400 \mathrm{~mL}$ or $70 \%$ of the programmed Last Fill Volume. ${ }^{6}$

The pneumatic forces may cause abdominal discomfort resulting in patients switching to TPD, CAPD, or other dialysis modalities. ${ }^{7}$ A recent Canadian survey of 293 APD patients suggests that drain pain may have been of significant severity and directly responsible for approximately one fourth of patients in this survey switching to TPD. ${ }^{8}$ This survey population of 293 APD patients was estimated as $15 \%$ of the PD patient population in Ontario, Canada. ${ }^{8}$ The drain pain was attributed to the negative pressure applied to the peritoneal membrane or negative suction to the bowel wall. In the case of TPD, these forces may be dispersed or diffused, with the residual solution acting as a buffer between the patient's peritoneum and bowel wall. ${ }^{7}$ However, although TPD may reduce "drain pain", it may also increase the risk of overfill. ${ }^{11}$

\section{Alarms}

While the purpose of alarms that were built into the cyclers is to ensure patient safety and use of proper technique, they can sometimes pose challenges for the patients and staff. Our survey results reveal that patients and nurses feel that at times, the cycler alarms are too generic. Examples of such alarms include low UF, kinked line, without specifying which connection or line is kinked; some alarms can be misleading such as "check patient line", which can be triggered by kinked drain line, closed clamp as well as kinked patient line. In some instances, patients who are "dry" during the day will trigger the "low drain volume alarm" when the cycler tries to perform the I-Drain. Other staff indicated that at times the alarms continue to beep for an extended period, and can be time consuming to investigate and resolve.

Teaching patients to find and address the exact problem for each alarm remains an ongoing process. Both Fresenius and Baxter manuals dedicate detailed sections to troubleshooting of alarms. Although patients are provided with 
these manuals to guide them in addressing these alarms, most patients tend to look at the display screen for guidance rather than refer to the large manual. If future models of the cyclers incorporated features of more specific alarm trouble shooting and guiding patients via the display screen, it would certainly make the cycler even more user friendly.

\section{Data entry}

Numerical data are entered using arrow keys to adjust values. Staff often note that the numbers/numerical data have a tendency to jump ahead during this process, consuming excessive time in programming data. An optional, compact keyboard for data entry could be a more user friendly and feasible future alternative. Some of our survey participants also suggested that the "sensitivity of touch" on the cycler might be an opportunity for improvement in the future, and this may address the tendency of the numbers to jump ahead while entering data such as the patient's weight and blood pressure.

\section{Challenges perceived by patients}

Many patients with end-stage renal disease are elderly, and the level of their technical education and experience varies widely. "Technophobia" was interestingly listed as a challenge encountered by many of the elderly cycler patients. Some may initially perceive the cycler as being complicated, and are doubtful regarding their ability to learn to perform CCPD. These patients need reassurance about the ease of use of the newer cycler models, its advantages, the support system available via the dialysis unit, on-call PD nurses, and help lines provided by Baxter and Fresenius. We find that the first point of contact for our patients is usually the PD nurse on-call who is able to guide/troubleshoot most of their problems, or direct them to the help lines if he/she is unable to fix the problem.

We propose a future opportunity for development to be the capability of remote live access to cyclers. With this advancement, the PD nurses or technical support staff could log in and remotely access the patient's individual cycler at home, or in the hospital, and assist patients in trouble shooting alarms when they may not be able to resolve the alarm on their own. This would be particularly comforting for the elderly patients who may be initially fearful. Although help-lines and on call PD nurses are currently available as mentioned, live support would be a stronger backup for these patients.

Baxter already offers a feature of phone line and modem use to transfer therapy information on the Pro Card to the dialysis unit, hence remote live access of the cycler may be the next step in development for future cyclers.

\section{Challenges faced by nursing staff}

The need for re-training patients with reminders of key techniques and providing refreshers in sterile techniques during connections was a common need among many of the centers we surveyed.

Additionally, despite home visits, staff are unable to mock a full home treatment and have to teach in sections, while trying to fast forward through sections they are not focusing on at that time. This may make it difficult for the learner, and it can take up to a week of performing CCPD at home before it all comes together for the patients. So a quick "re-training" session may also become necessary here.

\section{Company support}

Both Baxter and Fresenius provide help lines available to patients and staff. We have received good feedback in our survey from the nursing staff, about the support provided to patients via these help lines, where company representatives are able to assist with trouble-shooting problems. As mentioned before, both companies also provide manuals or patient guides; however, feedback from staff note some parts of these manuals to be "wordy" and confusing in terms of the terminology used for patients.

\section{Transitioning from CCPD to CAPD}

Although few patients make this transition, we wanted to explore what factors contribute to this decision. One common reason appears to be a persistently positional catheter that will not drain unless the patient sits or stands. This causes the cycler to alarm every time it attempts to drain unsuccessfully while the patient is asleep. We have one case where the patient sets an alarm clock and awakens to sit up in bed to allow the cycler to drain with each exchange. Of course, this is a tedious and impractical choice for many other individuals and has resulted in patients preferring to perform CAPD during the day. Other patients opt to switch due to a variety of reasons, including persistent drain pain despite attempting TPD, anxiety of being tethered/attached to cycler for prolonged duration of time overnight, frequent alarms, portability, duration of therapy, and difficult work schedules.

\section{Digital card}

Both Fresenius and Baxter models now provide the feature of a digital card that can store dialysis therapy information for patients varying from 30 days to 90 days, depending on the cycler model and size of the card. The dialysis unit can then download this information during the monthly visits. The digital cards are certainly a convenient and efficient 
method of transferring therapy details and work well for many patients. However, there have been occasional problems encountered with the digital cards where at times some therapies and, at other times, complete therapy information is deleted or missing on reliable patients. In addition, some patients forget to bring their Pro Cards to their monthly visits at times. Here, the Baxter feature of therapy transfer via a modem and phone line can be a key solution.

\section{Conclusion}

Our goal in this review is to provide a better understanding of today's PD cycler, including its technical aspects and practical uses. The cycler is a major advancement in the field of PD and has improved the convenience and efficiency of PD for a large number of patients. Both Baxter and Fresenius continue to work on technical advancements, and newer models of the cycler are in the development phase, to be available in the near future.

\section{Acknowledgments}

We are indebted to Drs Joel Glickman, Eric Wallace, Joanne Bargman, and John Burkart, and nurses Chloe Harkins, Stefan Trohonel, Joan Leake-Nardeo, Josefina Nunez, Alicia Zerkle, Ann Schatzkin, Carrie Nelson, Gayla East, Leanne Dean, Margaret Beaver, Sherry Hamilton, and Susan Vann for their significant contributions. We would also like to acknowledge Dr Mary Gellens, Lisa Alles and Aaron Brown at Baxter, and Helene Olefsky at Fresenius, for providing technical information.

\section{Disclosure}

The authors report no conflicts of interest in this work.

\section{References}

1. Ganter G. Uber die beseitigung giftiger stoffe aus dem blute durch dialyse. Munvpch Med Wschr. 1923;70:1478-1480.

2. Palmer R. As it was in the beginning: a history of peritoneal dialysis. Perit Dial Int. 1982;2(1):16-22.

3. Jain AK, Blake P, Cordy P, Garg AX. Global trends in rates of peritoneal dialysis. J Am Soc Nephrol. 2012;23(3):533-544.

4. Nolph D. Periotneal Dialysis. 3rd ed. Dordrecht, The Netherlands: Kluwer Academic Publishers; 1989.

5. Oreopoulos DG, Thodis E. The history of peritoneal dialysis: Early years at Toronto Western Hospital. Dial Transplant. 2010;39(8):338-343.

6. Baxter. HomeChoice and HomeChoice Pro APD Systems Trainer's Guide. Deerfield: Baxter; 2012.

7. Blake P. Drain pain, overfill, and how they are connected. Perit Dial Int. 2014;34(4):342-344.

8. Blake PG, Sloand JA, McMurray S, Jain AK, Matthews S. A multicenter survey of why and how tidal peritoneal dialysis (TPD) is being used. Perit Dial Int. 2014;34(4):456-458.

9. Baxter. The HomeChoice and HomeChoice Pro APD Systems Patient At-Home Guide; 2009. Available from: http://www.baxter. com/downloads/patients_and_caregivers/products/10.21_FINAL_ VERSION_PAHG_071961244.pdf. Accessed November 29, 2014.

10. Fresenius Medical Care. Operator's Manuals. Available from: http:// www.fmcna.com/fmcna/operatorsmanuals/operators-manuals.html. Date unknown. Accessed November 29, 2014.

11. Davis ID, Cizman B, Mundt K, et al. Relationship between drain volume/fill volume ratio and clinical outcomes associated with overfill complaints in peritoneal dialysis patients. Perit Dial Int. 2011;31(2):148-153. 


\section{Supplementary material}

\section{Survey for PD nurses}

- What three problems do you face most frequently in operating the cycler?

- What would you change in the cycler if you could?

- What are the three major challenges in training the patients to use the cycler?

- How helpful is the Baxter/Fresenius troubleshoot line in answering questions?

- What three complications do you feel patients develop most on the cycler?
- Why do patients transition off cycler to manual PD?

- What are your thoughts/experiences on the cycler alarms - nuisance at times or well programmed and mostly appropriate?

- Have you had problems with transferring data from the Pro Card to your operating system? Does the company do a good job of updating their operating system/programming to keep up with new windows software and other operating systems?

\section{Publish your work in this journal}

Medical Devices: Evidence and Research is an international, peerreviewed, open access journal that focuses on the evidence, technology, research, and expert opinion supporting the use and application of medical devices in the diagnosis, treatment and management of clinical conditions and physiological processes. The identification of novel devices and optimal use of existing devices which will lead to improved clinical outcomes and more effective patient management and safety is a key feature. The manuscript management system is completely online and includes a quick and fair peer-review system. Visit http://www. dovepress.com/testimonials.php to read real quotes from authors. 